\title{
Effects of Garlic Extract, Anti-Estrogens, and Aromatase Inhibitor on Sex Differentiation in Embryo
}

\author{
Einollah Valizadeh ${ }^{1}$, Hamed Seratinouri ${ }^{1 *}$
}

\section{Article History:}

Received April 2013

Accepted May 2013

Available online July 2013

\section{Keywords:}

Aromatase Inhibitor

Anti-estrogen

Sex-reversed Chicken

\section{Corresponding Author:}

Hamed Seratinouri, Drug Applied

Research Center, Student

Research Committee Tabriz university of Medical Sciences,

Tabriz, Iran.

Tel: +989144001704

Email: hamed.serati@gmail.com

\begin{abstract}
Objective: to investigate the effects of administrations of aromatase inhibitor and anti-estrogens on sex differentiation.

Material and Methods: Eggs were divided into five groups of 60 , one control group receiving distilled water and four test groups. All groups were placed inside the incubator. Injection of anti-aromatases was performed in fifth day of incubation. First test group received $1 \mathrm{mg} / \mathrm{egg}$ aromatase inhibitor (AI), second test group received $1 \mathrm{mg} /$ egg Tamoxifen, third test group received $1 \mathrm{mg} /$ egg Clomiphene and forth test group received 1mg/egg GAR79 respectively. Chickens were determined after hatching on first day using Feather Sexing method. Redetermination was performed after 45 days for more insurance. In this study 14- $\alpha$-hydroxy 3,6,17, androstantrion inhibitor with dose of $1 \mathrm{mg} /$ egg were injected.

Results: Administration of anti-aromatases into the eggs during incubation period causes production of male fetus about $\% 72$ to $\% 84$ that in comparison with control group (\%52/5 male) showed an increase of \%20 to \%34 (P $\geq 0.05$ ). The sex reversed females have bilateral testes with ability of complete spermatogenesis and also the physical appearance and behavior of normal males.

Conclusion: Results showed the significant role of aromatase in sex differentiation. Moreover, results showed GAR79 as an anti-aromatase could affect aromatase activity in sex differentiation.
\end{abstract}

1. Drug Applied Research Center, Student Research Committee, Tabriz university of Medical Sciences, Tabriz, Iran. 


\section{Introduction:}

Sex differentiation is a regular and consecutive process. Egg genotype determines the nature of sex glands, which then lead to evolution of egg to male or female (1). In mammals, Y chromosome has an important role in testicular evolution from undifferentiated gonads. Testis development and sex differentiation depends more on Anti-Müllerian hormone (AMH) and androgens secreted by testes (2). AMH secreted by Sertoli cells prevents development of Müllerian duct and subsequently female reproductive system (3). Levels of steroid hormones were regulated by inhibiting of aromatase enzyme, which catalyzes the last stage of sex steroid biosynthesis route and convert androgens into estrogens (1). There are 35 kilo base pairs on the $\mathrm{Y}$ chromosome before the end of a pseudo-autosomal region that encodes a polypeptide of 233 amino acids. This area is called SRY gene or (sex determining region of $\mathrm{Y}$ chromosome) (4). Other genes called SOX family has been identified which encode proteins that have more than $60 \%$ similarity with the SRY HMG box. SOX9 has an important role in sex determination (5). In the ZZ-ZW system, female chickens are heterogametic (ZW) while male chickens are homogametic (ZZ) and their SRY are automatically non-similar. W chromosome like Y chromosome has an important role in sex-determination. In birds, sex steroids and anti-Mullerian hormone naturally takes part in sex differentiation. Furthermore, estrogen and androgen have important role on female and male production respectively. Aromatase P450 (P450 arom) is known as a key enzyme in biosynthesis of estrogen and expression of P450 arom mRNA is earliar in female chicks than AMH mRNA expression in males. Therefore, $\mathrm{P} 450$ arom gene expression is as an important process in the beginning of female distinction. This phenomenon is directly associated with genetic sex-determination (6).

Immunohistochemical studies showed that gender depends on different amounts of androgen and estrogen production after day 5 / 6 of incubation. The production of androgens in male gonads is more than female gonads whereas the production of estrogen in female gonads is more than male gonads (7). Embryo chicks of both sexes have the ability of androgen synthesis whereas embryos with female genotypes could produce estrogen apart from androgen during short period before the gender distinction of gonads. Therefore, in female chicken embryo P450 arom mRNA expression has an important role in the early stages of estrogen production. Another study on rat brain showed that testosterone is effective on the brain development to determine the Genderspecific capacity for aromatization and to regulate androgen responsiveness within components of the neural circuitry that mediates male sexual behavior (10). Plants contain some essential phytochemicals produced for their various vital functions (11). In addition to being a source of compounds necessary for human nutrition, certain plant foods also contain compounds that may have long-term effects on human and animal health. Among these compounds, the most important are the phytoestrogens. (12) Phytoestrogens naturally share with steroidal estrogens an ability to activate estrogen receptors (11). There are several excellent reviews documenting and evaluating the large and complex body of evidences demonstrating the healthy and beneficial effects of phytoestrogens for humans and laboratory animals (13-17). Phytoestrogens divided into several groups such as isoflavonoids, flavonoids and lignans. Garlic is one of dietry source of lignans and major phytoestrogen compounds of garlic are secoisolariciresinol and Matairesionol. (11) The purpose of this study was evaluation the effects of aromatase inhibitors estradiol 14 - alpha - hydroxy -3, 6, 17 Androstenetrione and two other antiestrogens named Tamoxifen, Clomiphene citrate, and garlic extract (GAR79) on sexdetermination of Arian broilers.

\section{Material \& Methods:}

In this study eggs from Arian race hens was used. Eggs divided into 5 groups of 60 , one control group and four test groups. All 
incubation, control group received $0.1 \mathrm{ml}$ distilled water, first test group received $1 \mathrm{mg}$ AI (donated by Professor Makoto Yoshihama from Strategic Research Institute of Japan), second test group received $1 \mathrm{mg}$ of Tamoxifen dissolved in water, third test group received $1 \mathrm{mg}$ Clomiphene citrate dissolved in water and forth test group received 1mg GAR79 (garlic extract compound which was formerly used in this study) respectively. Injections were performed from the small and thin end of eggs (in front of air space) using insulin syringe into egg white. Thereafter, Egg holes were closed with melted paraffin blocks and eggs were returned to the incubation device. The sexes Chickens were determined after hatching on first day using Feather Sexing method. Redetermination was performed after 45 days for more insurance.

Statistical analysis :

The results were analyzed by performing chi square test. P-value of $<0.05$ was considered statistically significant.

\section{Results:}

The results were shown in the following figure (see figure 1). In all four experimental groups, there were significant changes in the number of male chicks. Tamoxifen and Clomiphene have negative effects in the rate of hatching of eggs. Even chickens treated with Clomiphene died in the first week of life. Macroscopic observations of genital system showed that in some cases ovaries became smaller in size and some others had medium size. Moreover, certain anomalies attributed to treatments were not found among chickens. This means that the injection of these agents change only the balance of sex steroid hormones at the time of sexdifferentiation.

Initial results from the effects of GAR79 extraction (which were used in this study as a plant extract) showed that with optimization of injection amount and completion of extraction process, this agent can be used as an anti-aromatase.

\section{Discussion:}

Since the chicken sex differentiation occurs in day 5 / 6 of incubation, any change in the concentration of sex hormones at this stage lead to changes in tissue structure of sex glands (2). AI with blocking aromatase activity increases androgens but decreases estrogen levels simultaneously (1) and antiestrogens with blocking of estrogen receptors prevent of estrogen effects. Thus androgen to estrogen ratio would be changed and sex-differentiation would be tended to male more than female gender (8). This study shows the dependence of sex- differentiation on sex hormones. Injection of estradiol benzoate or aromatase inhibitors has no effect on the general conditions of fetus. The basis of sensitivity of sex-differentiation to estrogen is unknown (8). Regulation of aromatase may be efficient in the sex-distinction of other species, for example defects in embryonic aromatase enzyme activity leads to pseudo hermaphroditism in female human embryo (9). The results of this study implies that regulation of aromatase activity as a main factor in controlling sex determination and these results are coordinated with the results of studies on other animal species $(1,2,3)$.

\section{Conclusion :}

According to the results, there is a significant role of aromatase in normal sex differentiation, so Anti-aromatases affect sex differentiation and change the ratio of male/female. Moreover, results confirmed GAR79 as an anti-aromatase could affect aromatase activity in sex differentiation.

\section{Conflict of interest statement:}

We declare that we have no conflict of interest.

Figure1. Comparison of the effects of various anti-aromatases on the percentage of production of male and female chickens

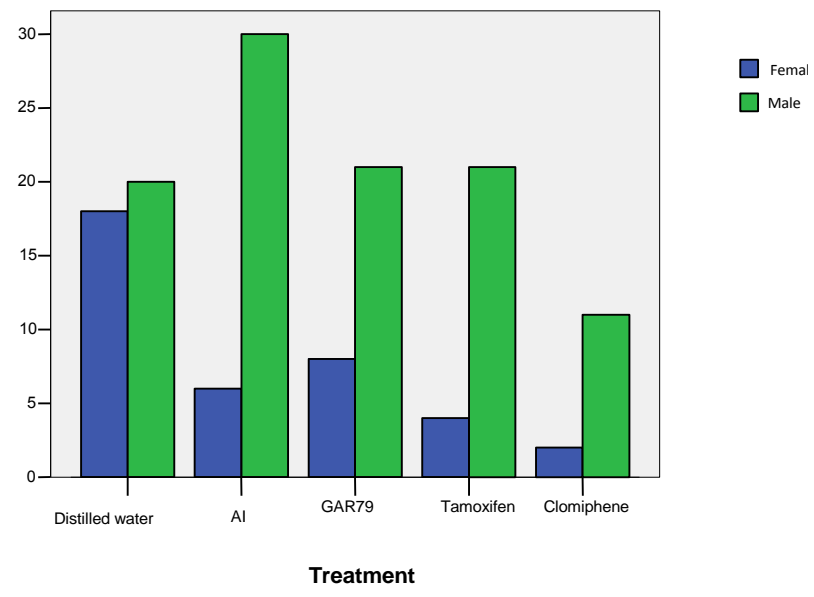




\section{References:}

1. Smith RG, Elbrecht A. Aromatase Enzyme Activity and sex Determination in chickens. Science. 1992; 255(5043): 467-70.

2. Shimada K, Saito N. Molecular Mechanisms of sex determination and sex diffrentiation. Japanese Poultry Science. 2000; 37: 3-11.

3.WibbelsT, et al. Steroid hormone-induced male sex determination is anaminiotic vertebrate.J Exp Zool. 1992; 262(4): 454-457.

4. Sincair AH, et al. Agene from the human sex-determining region encodes a protein with homology to a conserved DNA-binding motif. Nature. 1990; 346(6281): 240-244

5. Kwok C, et al. Mutations in sox9, the gene responsible for campomelic dysplasia and autosomal sex reversal. Am J Hum.Genet. 1995; 57(5):1028-1036

6. Shimada K. Gene expression of steroidogenic Enzymes in chicken embryonic Gonads. J Exp Zool. 1998; 281(5): 450-6

7. Smith CA, et al. Gondadelsen differentation in chicken emberyos. Experssion of estrogenreceptor and aromatase gens. J Steroid Bichem Mol Biol. 1997; 60: 295-302.

8. Baltazartj, et al. Behavioral Demasculinzation of Female quail is induced by estrogens: Studies with new Aromatase inhibitor, R76713 Hormones and Behavior. 1992; 26(2): 179203.

9. Shozu, M, Akasofu K, Harada T, Kubota Y. A new cause of female Pseudohermaphroditism: placental aromatase deficiency J Clin Endocrinol Metab. 1991; 72: 560-566.

10. Roselli CE, Klosterman SA, Sexual differentiation of aromatase activity in the rat brain: effects of perinatal steroid exposure. Endocrinology. 1998; 139(7): 3193-201.

11. Mazur, W.Phytoestrogen content in foods. Baillieres Clin Endocrinol Metab. 1998; 12(4): 729-742.

12. Dixon RA. Phytoestrogens. Annu. Rev. Plant Biol. 2004; 55: 225-261.

13. Adlercreutz H. Human Health and Phytoestrogens. Reproductive Toxicology and Development. 1998; 299-371.

14. Adlercreutz H. Phyto-oestrogens and cancer. Lancet Oncol. 2002; 3(6): 364-73

15. Adlercreutz H, Mazur W. Phytoestrogens and Western diseases. Ann. Med. 1997; 29:95120 
16. Adlercreutz M. Epidemiology of phytoestrogens. Baillieres Clin Endocrinol Metab. 1998; 12: $605-23$

17. Setchell KDR, Cassidy A. Dietary isoflavones: biological effects and relevanceto human health. J Nutr. 1999; 129(3):758S-67S 